\title{
The Effects on Parameter Estimation of Correlated Dimensions and a Distribution-Restricted Trait in a Multidimensional Item Response Model
}

\author{
Rose-Marie Batley and Marvin W. Boss
}

The University of Ottawa

\begin{abstract}
This study was designed to assess the effects on parameter estimation of correlated dimensions and a distribution-restricted trait on one dimension using a two-dimensional item response theory model. Multidimensional analysis of simulated two-dimensional item response data fitting a multidimensional two-parameter logistic item response theory model (McKinley \& Reckase, 1983a; Reckase \& McKinley, 1991) was done using the program MIRTE (Carlson, 1987). Six datasets ( 2 trait distributions $\times 3$ levels of correlation between dimensions) of 2,000 trait vectors over 104 items were generated. Each dataset was analyzed and replicated 100 times. Trait and item parameters generally were recovered adequately in the datasets in which both traits were normally distributed over the full range. In the datasets with a restricted range of trait level on the second dimension, recovery of the trait and item parameters was affected adversely. The results indicated that MIRTE recovers the structure of a multidimensional correlated space better than reported in earlier studies, especially when items are multidimensional. Index terms: correlated traits, multidimensional item parameter estimates, multidimensional item response theory, multidimensional trait estimates, restricted traits.
\end{abstract}

Early item response theory (IRT) models were based on the assumption of unidimensionality (i.e., only one trait is required to respond correctly to all items). When more than one trait accounts for test performance, the test is multidimensional and a multidimensional IRT (MIRT) model is appropriate.

Several researchers (Ackerman, 1987; Ansley

APPLIED PSYCHOLOGICAL MEASUREMENT

Vol. 17, No. 2, June 1993, pp. 131-141

(C) Copyright 1993 Applied Psychological Measurement Inc.

0146-6216/93/020131-11\$1.80
\& Forsyth, 1985; Bogan \& Yen, 1983; Dorans \& Kingston, 1985; Drasgow \& Parsons, 1983; McCauley \& Mendoza, 1985; McKinley \& Reckase, 1984; Reckase, 1979, 1985; Reckase, Carlson, Ackerman, \& Spray, 1986) have analyzed known multidimensional data with a unidimensional item response model. Unidimensional trait estimates have been found to be related to the average of the multidimensional traits (Ansley \& Forsyth, 1985); unidimensional trait estimates also have been found to have different interpretations at different points on the unidimensional trait scale (Reckase et al., 1986). In general, unidimensional estimates from multidimensional data have been difficult to interpret and have not reflected well the original characteristics of the data.

Researchers who have used multidimensional IRT analysis (e.g., McKinley, 1983; McKinley \& Reckase, 1983a, 1983b, 1984; Muraki \& Englehard, 1985) have indicated that a multidimensional model more adequately describes both real and simulated multidimensional data than does a unidimensional model. However, because most of the simulation studies have not used replications, the stability of the estimates is difficult to determine. It is necessary to know how consistently these estimates are recovered.

Consider the situation in which items for a test are designed to measure one trait (e.g., mathematics ability) but require some amount of a second trait (e.g., verbal ability) for the examinee to respond correctly. This second required trait could be more crucial to success for some 
examinees than others. Students of English as a second language (ESL) may have sufficient mathematics ability but lack the required amount of verbal ability to respond correctly.

It is reasonable to assume that the two traits are correlated. Therefore, it is appropriate to evaluate the effects of both correlated traits and a secondary trait, which is restricted in its distribution, on parameter estimation.

\section{Purpose}

This study was designed to determine the adequacy of multidimensional trait and item parameter estimates using a MIRT analysis. Three questions were addressed:

1. What is the effect of correlated trait dimensions on parameter estimation for a twoparameter, two-dimensional IRT model when both traits are normally distributed?

2. How is parameter estimation affected by a restricted secondary trait?

3. Are the effects of correlated dimensions similar when both traits are normally distributed, and when a restricted trait exists on the secondary dimension?

\section{Method}

\section{Model Description}

The data were generated to fit a multidimensional two-parameter logistic (M2PL) IRT model (McKinley \& Reckase, 1983a; Reckase \& McKinley, 1991). The M2PL model is defined as

$P_{i j}=P\left(X_{i j}=1 \mid \mathbf{a}_{i}, d_{i}, \boldsymbol{\theta}_{j}\right)=\frac{\exp \left(\mathbf{a}_{i} \boldsymbol{\theta}_{j}+d_{i}\right)}{1+\exp \left(\mathbf{a}_{i} \boldsymbol{\theta}_{j}+d_{i}\right)},(1)$

$(i=1,2, \ldots, n ; j=1,2, \ldots, N)$

where

$P_{i j}$ is the probability of a correct response to item $i$ by examinee $j$;

$X_{i j}$ is the response $(1=$ correct; $0=$ incorrect $)$ of examinee $j$ to item $i$;

$\mathbf{a}_{i}$ is a vector of $m$ discrimination parameters (where $m$ is the number of dimensions);

$d_{i}$ is a parameter representing the difficulty of item $i$

$\boldsymbol{\theta}_{j}$ is a vector of $m$ trait parameters for individual $j$;

$N$ is the number of examinees; and

$n$ is the number of items.

This model is compensatory-it allows high levels on one dimension to compensate for low levels on other dimensions in arriving at a correct (or keyed) response to an item.

Reckase \& McKinley (1991) defined a multidimensional discrimination parameter for item $i$ as:

$A_{i}=\left[\sum_{k=1}^{m}\left(a_{i k}\right)^{2}\right]^{1 / 2}$.

[Reckase \& McKinley (1991) originally defined multidimensional discrimination as MDISC, rather than $A$.] $A$ is proportional to the slope of the item response function at the point of steepest slope and it is, therefore, analogous to the unidimensional discrimination parameter (Carlson, 1987).

Reckase (1985) and Reckase \& McKinley (1991) also defined a multidimensional item difficulty parameter, $D_{i}$, as:

$D_{i}=-d_{i} / A_{i}$.

[Reckase (1985) and Reckase \& McKinley (1991) defined multidimensional item difficulty as MDIF, rather than $D$.] $D$ represents the distance between the origin of the $m$-dimensional trait space and the point in the space at which the item information is a maximum. The line joining this point to the origin is at an angle of $\alpha_{i k}$ to the $k$ th trait dimension where

$\cos \alpha_{i k}=a_{i k} / A_{i}$.

\section{Data}

Tests and responses. Six datasets were used. Datasets 1, 2, and 3 represented cases in which both underlying traits $\left(\theta_{1}\right.$ and $\left.\theta_{2}\right)$ were normally distributed with mean 0 and standard deviation (SD) 1 . These datasets differed in the degree of correlation between $\theta_{1}$ and $\theta_{2}$, which was $0.00, .25$, and .50 , respectively. In 
Datasets 4,5 , and $6, \theta_{1}$ was normally distributed (mean $=0, \mathrm{SD}=1$ ), but $\theta_{2}$ was normally distributed with a lower mean and SD ( -1 and .67, respectively). These datasets also varied in the degree of correlation $(0.00, .25$, and .50 , respectively).

The simulated test contained 104 items-26 items required only $\theta_{1}$ (i.e, only the first dimension had a nonzero discrimination), 52 items required predominantly $\theta_{1}$, and 26 items required approximately equal amounts of $\theta_{1}$ and $\theta_{2}$. The item parameters are provided in Table 1 . To meet the requirement that the items discriminate well on $\theta_{1}$, four values of the angle, $\alpha_{i 1}$, were selected $-0^{\circ}, 15^{\circ}, 30^{\circ}$, and $45^{\circ} .13$ values of $D$ (ranging from -3 to +3 at intervals of .5 ) were selected to cover the range of difficulties; two values of $A(2.00,1.70)$ were selected to simulate realistic discrimination conditions in which the items were designed to discriminate well on $\theta_{1}$. $a_{1}$ and $a_{2}$ (one for each dimension) then were generated to fit the corresponding $d_{i}$ and $A .104$ items may be considered a long test, but it was necessary in order to have an item at each of the levels of $\alpha_{i 1}, D$, and $A$. The correlations between these item parameters were: $\rho\left(d, a_{1}\right)=.004$; $\rho\left(d, a_{2}\right)=-.004 ; \rho\left(a_{1}, a_{2}\right)=-.738 ;$ and $\rho(D, A)$ $=-.002$. Because of the dependency of $a_{1}$ and $a_{2}$, there was a larger correlation between $a_{1}$ and $a_{2}$. The same item parameters were used for each of the six datasets.

Analysis. Program M2PLGEN (Ackerman, $1985)$ was used to generate $2,000 \theta$ vectors $\left(\theta_{1}, \theta_{2}\right)$ satisfying the specified distributions of $\theta_{1}$ and $\theta_{2}$ for each dataset. M2PLGEN uses a random seed and the International Mathematical and Statistical Libraries (1979) subroutine GGNSM to generate random $\theta$ levels. These $\theta$ vectors and the item parameters $\left(a_{1}, a_{2}, d\right)$ then were used to generate response vectors ( $0 \mathrm{~s}$ and $1 \mathrm{~s})$ to each item for the 2,000 simulees according to the M2PL model. At the end of each replication, the random seed used by M2PLGEN to generate $\theta$ response vectors was increased by 2 and the process was repeated.

Program MIRTE Version 2.0 (Carlson, 1987) was used to analyze the two-dimensional data.
MIRTE estimated $\theta \mathrm{s}, a \mathrm{~s}$, and $d \mathrm{~s}$ for the M2PL model, estimates of the standard errors (SES) for each of these parameter estimates, and estimates of $A$ and $D$. The estimation method used is a variation of the joint maximum likelihood procedure using a modified Newton-Raphson iteration technique. The algorithm used is similar to that used in the unidimensional analysis program LOGIST (Wingersky, Barton, \& Lord, 1982).

For each replication, the $2,000 \times 104$ matrix of response vectors was analyzed using MIRTE. The initial item parameter estimates for $a_{1}$ and $a_{2}$ were randomly generated according to the requirements of MIRTE: MIRTE requires that they be any positive number less than the maximum value defined by the user for the discrimination parameters, in this case 2.0. The same estimates were used for every replication and in every dataset to provide better control. Initial difficulty parameter estimates were computed by MIRTE. Means and SDs of $\hat{\theta}_{1}, \hat{\theta}_{2}$, the number-correct score, and item parameter estimates were calculated for each replication. For each parameter estimate, the average absolute deviation (AAD) the average absolute deviation of the estimate from the true value of the variable-also was calculated.

Means of the SEs of $\hat{\theta}_{1}, \hat{\theta}_{2}, \hat{d}, \hat{a}_{1}$, and $\hat{a}_{2}$ also were determined, and correlations between parameters and estimates were calculated. Mean statistics then were calculated over the 100 replications for each of the six datasets.

\section{Results}

\section{Adequacy of the Simulated Data}

To assess the effects on parameter estimation of correlated $\theta$ s and a restricted $\theta$ on one dimension, it was necessary to determine if suitable $\theta$ s were generated to model the conditions specified. It was also important to determine whether MIRTE adequately estimated the parameters of the response vectors generated.

For Datasets $1-3$, the $\theta$ vectors were both normally distributed over a full range. The correlation between $\theta_{1}$ and $\theta_{2}$ for data generated over 
Table 1

True Item Parameters for the 104 Items

\begin{tabular}{|c|c|c|c|c|c|c|c|c|c|c|}
\hline$\alpha_{11}$ & $D_{\imath}$ & $d_{t}$ & $\begin{array}{c}\text { Item } \\
\text { Number }\end{array}$ & $A$ & $a_{t 1}$ & $a_{t 2}$ & $\begin{array}{c}\text { Item } \\
\text { Number }\end{array}$ & $A$ & $a_{11}$ & $a_{i 2}$ \\
\hline $0^{\circ}$ & 3.0 & -6 & 1 & 2.00 & 2.00 & 0.00 & 53 & 1.70 & 1.70 & 0.00 \\
\hline $0^{\circ}$ & 2.5 & -5 & 2 & 2.00 & 2.00 & 0.00 & 54 & 1.70 & 1.70 & 0.00 \\
\hline $0^{\circ}$ & 2.0 & -4 & 3 & 2.00 & 2.00 & 0.00 & 55 & 1.70 & 1.70 & 0.00 \\
\hline $0^{\circ}$ & 1.5 & -3 & 4 & 2.00 & 2.00 & 0.00 & 56 & 1.70 & 1.70 & 0.00 \\
\hline $0^{\circ}$ & 1.0 & -2 & 5 & 2.00 & 2.00 & 0.00 & 57 & 1.70 & 1.70 & 0.00 \\
\hline $0^{\circ}$ & .5 & -1 & 6 & 2.00 & 2.00 & 0.00 & 58 & 1.70 & 1.70 & 0.00 \\
\hline $0^{\circ}$ & 0.0 & 0 & 7 & 2.00 & 2.00 & 0.00 & 59 & 1.70 & 1.70 & 0.00 \\
\hline $0^{\circ}$ & -.5 & 1 & 8 & 2.00 & 2.00 & 0.00 & 60 & 1.70 & 1.70 & 0.00 \\
\hline $0^{\circ}$ & -1.0 & 2 & 9 & 2.00 & 2.00 & 0.00 & 61 & 1.70 & 1.70 & 0.00 \\
\hline $0^{\circ}$ & -1.5 & 3 & 10 & 2.00 & 2.00 & 0.00 & 62 & 1.70 & 1.70 & 0.00 \\
\hline $0^{\circ}$ & -2.0 & 4 & 11 & 2.00 & 2.00 & 0.00 & 63 & 1.70 & 1.70 & 0.00 \\
\hline $0^{\circ}$ & -2.5 & 5 & 12 & 2.00 & 2.00 & 0.00 & 64 & 1.70 & 1.70 & 0.00 \\
\hline $0^{\circ}$ & -3.0 & 6 & 13 & 2.00 & 2.00 & 0.00 & 65 & 1.70 & 1.70 & 0.00 \\
\hline $15^{\circ}$ & 3.0 & -6 & 14 & 2.00 & 1.932 & .518 & 66 & 1.70 & 1.642 & .44 \\
\hline $15^{\circ}$ & 2.5 & -5 & 15 & 2.00 & 1.932 & .518 & 67 & 1.70 & 1.642 & .44 \\
\hline $15^{\circ}$ & 2.0 & -4 & 16 & 2.00 & 1.932 & .518 & 68 & 1.70 & 1.642 & .44 \\
\hline $15^{\circ}$ & 1.5 & -3 & 17 & 2.00 & 1.932 & .518 & 69 & 1.70 & 1.642 & .44 \\
\hline $15^{\circ}$ & 1.0 & -2 & 18 & 2.00 & 1.932 & .518 & 70 & 1.70 & 1.642 & .44 \\
\hline $15^{\circ}$ & .5 & -1 & 19 & 2.00 & 1.932 & .518 & 71 & 1.70 & 1.642 & .44 \\
\hline $15^{\circ}$ & 0.0 & 0 & 20 & 2.00 & 1.932 & .518 & 72 & 1.70 & 1.642 & .44 \\
\hline $15^{\circ}$ & -.5 & 1 & 21 & 2.00 & 1.932 & .518 & 73 & 1.70 & 1.642 & .44 \\
\hline $15^{\circ}$ & -1.0 & 2 & 22 & 2.00 & 1.932 & .518 & 74 & 1.70 & 1.642 & .44 \\
\hline $15^{\circ}$ & -1.5 & 3 & 23 & 2.00 & 1.932 & .518 & 75 & 1.70 & 1.642 & .44 \\
\hline $15^{\circ}$ & -2.0 & 4 & 24 & 2.00 & 1.932 & .518 & 76 & 1.70 & 1.642 & .44 \\
\hline $15^{\circ}$ & -2.5 & 5 & 25 & 2.00 & 1.932 & .518 & 77 & 1.70 & 1.642 & .44 \\
\hline $15^{\circ}$ & -3.0 & 6 & 26 & 2.00 & 1.932 & .518 & 78 & 1.70 & 1.642 & .44 \\
\hline $30^{\circ}$ & 3.0 & -6 & 27 & 2.00 & 1.732 & 1.00 & 79 & 1.70 & 1.472 & .85 \\
\hline $30^{\circ}$ & 2.5 & -5 & 28 & 2.00 & 1.732 & 1.00 & 80 & 1.70 & 1.472 & .85 \\
\hline $30^{\circ}$ & 2.0 & -4 & 29 & 2.00 & 1.732 & 1.00 & 81 & 1.70 & 1.472 & .85 \\
\hline $30^{\circ}$ & 1.5 & -3 & 30 & 2.00 & 1.732 & 1.00 & 82 & 1.70 & 1.472 & .85 \\
\hline $30^{\circ}$ & 1.0 & -2 & 31 & 2.00 & 1.732 & 1.00 & 83 & 1.70 & 1.472 & .85 \\
\hline $30^{\circ}$ & .5 & -1 & 32 & 2.00 & 1.732 & 1.00 & 84 & 1.70 & 1.472 & .85 \\
\hline $30^{\circ}$ & 0.0 & 0 & 33 & 2.00 & 1.732 & 1.00 & 85 & 1.70 & 1.472 & .85 \\
\hline $30^{\circ}$ & -.5 & 1 & 34 & 2.00 & 1.732 & 1.00 & 86 & 1.70 & 1.472 & .85 \\
\hline $30^{\circ}$ & -1.0 & 2 & 35 & 2.00 & 1.732 & 1.00 & 87 & 1.70 & 1.472 & .85 \\
\hline $30^{\circ}$ & -1.5 & 3 & 36 & 2.00 & 1.732 & 1.00 & 88 & 1.70 & 1.472 & .85 \\
\hline $30^{\circ}$ & -2.0 & 4 & 37 & 2.00 & 1.732 & 1.00 & 89 & 1.70 & 1.472 & .85 \\
\hline $30^{\circ}$ & -2.5 & 5 & 38 & 2.00 & 1.732 & 1.00 & 90 & 1.70 & 1.472 & .85 \\
\hline $30^{\circ}$ & -3.0 & 6 & 39 & 2.00 & 1.732 & 1.00 & 91 & 1.70 & 1.472 & .85 \\
\hline $45^{\circ}$ & 3.0 & -6 & 40 & 2.00 & 1.414 & 1.414 & 92 & 1.70 & 1.202 & 1.202 \\
\hline $45^{\circ}$ & 2.5 & -5 & 41 & 2.00 & 1.414 & 1.414 & 93 & 1.70 & 1.202 & 1.202 \\
\hline $45^{\circ}$ & 2.0 & -4 & 42 & 2.00 & 1.414 & 1.414 & 94 & 1.70 & 1.202 & 1.202 \\
\hline $45^{\circ}$ & 1.5 & -3 & 43 & 2.00 & 1.414 & 1.414 & 95 & 1.70 & 1.202 & 1.202 \\
\hline $45^{\circ}$ & 1.0 & -2 & 44 & 2.00 & 1.414 & 1.414 & 96 & 1.70 & 1.202 & 1.202 \\
\hline $45^{\circ}$ & .5 & -1 & 45 & 2.00 & 1.414 & 1.414 & 97 & 1.70 & 1.202 & 1.202 \\
\hline $45^{\circ}$ & 0.0 & 0 & 46 & 2.00 & 1.414 & 1.414 & 98 & 1.70 & 1.202 & 1.202 \\
\hline $45^{\circ}$ & -.5 & 1 & 47 & 2.00 & 1.414 & 1.414 & 99 & 1.70 & 1.202 & 1.202 \\
\hline $45^{\circ}$ & -1.0 & 2 & 48 & 2.00 & 1.414 & 1.414 & 100 & 1.70 & 1.202 & 1.202 \\
\hline
\end{tabular}

continued on the next page 
R.-M. BATLEY and M. W. BOSS

PARAMETER ESTIMATION IN A MULTIDIMENSIONAL IRT MODEL

Table 1, continued

True Item Parameters for the 104 Items

\begin{tabular}{ccccccccccc}
\hline \hline & & \multicolumn{1}{c}{ Item } & & \multicolumn{7}{c}{ Item } \\
$\boldsymbol{\alpha}_{i 1}$ & $D_{i}$ & $d_{i}$ & Number & $A$ & $a_{i 1}$ & $a_{i 2}$ & Number & $A$ & $a_{i 1}$ & $a_{i 2}$ \\
\hline $45^{\circ}$ & -1.5 & 3 & 49 & 2.00 & 1.414 & 1.414 & 101 & 1.70 & 1.202 & 1.202 \\
$45^{\circ}$ & -2.0 & 4 & 50 & 2.00 & 1.414 & 1.414 & 102 & 1.70 & 1.202 & 1.202 \\
$45^{\circ}$ & -2.5 & 5 & 51 & 2.00 & 1.414 & 1.414 & 103 & 1.70 & 1.202 & 1.202 \\
$45^{\circ}$ & -3.0 & 6 & 52 & 2.00 & 1.414 & 1.414 & 104 & 1.70 & 1.202 & 1.202 \\
\hline
\end{tabular}

the 100 replications was .001 for Dataset $1, .251$ for Dataset 2, and .500 for Dataset 3. The means for $\theta_{1}$ and $\theta_{2}$ ranged from .002 to -.004 , and SDS were within $1 \pm .003$. For Datasets $4-6$, the correlations were equally well generated and similar results to Datasets 1-3 were found for $\theta_{1}(.001$ for Dataset 4, .251 for Dataset 5, and .499 for Dataset 6). For $\theta_{2}$, the means ranged from -.999 to -1.001 . The variance for the means and SDS for both $\theta_{1}$ and $\theta_{2}$ over the 100 replications was very small (less than .0005) in all datasets.

In agreement with the findings of Greaud (1988), the mean number of items correct appeared to be unaffected by changes in the degree of correlation between the $\theta$ dimensions. This may be because a compensatory model was used. For Datasets 1-3, all number-correct score means were approximately 52 . As expected, the means for Datasets 4-6 were lower (approximately 47) because of the lower mean $\theta_{2}$ values.

For all datasets over the 100 replications, $\hat{\theta}_{1}$ and $\hat{\theta}_{2}$ had means of 0.0 and SDs of 1.0. This is a function of MIRTE-it rescales the $\theta$ estimates to $\mathrm{N}(0,1)$ after each iteration. This presented problems for the estimation of item parameters for Datasets 4-6 because $\theta_{2}$ was generated as $\mathrm{N}(-1.0, .67)$.

MIRTE did not always identify Dimensions 1 and 2 correctly in the estimated results. To avoid incorrect identifications of dimensions during the 100 replications, the first 13 item discrimination parameter estimates for each replication were analyzed, because they were pure on $\theta_{1}$. If the sum of these $a_{1}$ estimates was less than the sum of the first $13 a_{2}$ estimates, the $\hat{a}$ s and $\hat{\theta}$ s for the estimates for the two dimensions were reversed (i.e., estimates identified as Dimension 1 were labeled as Dimension 2 and vice versa).

\section{Recovery of $\boldsymbol{\theta}$}

In Datasets 1-3, the degree of correlation did not seem to affect the recovery of $\theta_{1}$ (see Table 2 ). This is shown by the mean AADs. $\theta_{2}$ was better estimated (lower AADs) as correlations between $\theta$ s increased.

For Datasets $4-6$, both $\theta_{1}$ and $\theta_{2}$ were better estimated when $\theta$ s were not correlated. The large AADs for $\hat{\theta}_{2}$ are a result of the rescaling of $\hat{\theta}_{2}$ to a mean of 0 and a SD of 1 . The AADs were higher in Datasets 4-6 than Datasets 1-3. In each instance, the mean AADs were consistent over replications. The mean SEs of the $\hat{\theta}$ s were slightly higher for Datasets 4-6 (.287) than for Datasets 1-3 (.259).

Table 2 also shows the correlations of the $\theta$ s with their estimates, averaged over the 100 replications. For Datasets 3 and 6 (in which the $\theta$ s were correlated .5), there was poorer recovery of $\theta_{1}(.831$ and .744$)$ and better recovery of $\theta_{2}(.865$ and .721). When the $\theta$ s were not correlated (Datasets 1 and 4), the correlations between $\hat{\theta}$ and $\theta$ were considerably lower in Dataset 4 for both $\theta_{1}(.842$ in Dataset 1 vs. .773 in Dataset 4$)$ and $\theta_{2}$ (.764 in Dataset 1 vs. .517 in Dataset 4).

The correlation between $\theta_{1}$ and $\theta_{2}$ was not well recovered. For Datasets 1 and $4, \rho\left(\theta_{1}, \theta_{2}\right)$ was overestimated ( $r=.062$ and .147 , respectively). For the remaining datasets, $\rho\left(\theta_{1}, \theta_{2}\right)$ was underestimated (Table 2 ). These results agree with those reported by Carlson (1987).

It is interesting to note the high correlation between $\theta_{1}$ and $\hat{\theta}_{2}$. For Datasets 4-6, these correlations were higher that those of $\theta_{2}$ with $\hat{\theta}_{2}$. Clearly, higher correlations between dimensions 
Table 2

Means Over 100 Replications of Statistics for Estimated $\theta$

\begin{tabular}{ccccccccr}
\hline \hline Dataset & $\rho\left(\theta_{1}, \theta_{2}\right)$ & $\mathrm{AAD}\left(\hat{\theta}_{1}\right)$ & $\mathrm{AAD}\left(\hat{\theta}_{2}\right)$ & $r\left(\hat{\theta}_{1}, \hat{\theta}_{2}\right)$ & $r\left(\theta_{1}, \hat{\theta}_{1}\right)$ & $r\left(\theta_{2}, \hat{\theta}_{2}\right)$ & $r\left(\theta_{1}, \hat{\theta}_{2}\right)$ & $r\left(\theta_{2}, \hat{\theta}_{1}\right)$ \\
\hline 1 & 0.00 & .447 & .544 & .062 & .842 & .764 & .505 & -.295 \\
2 & .25 & .446 & .470 & .179 & .842 & .824 & .603 & -.050 \\
3 & .50 & .459 & .412 & .282 & .831 & .865 & .699 & .209 \\
4 & 0.00 & .463 & .856 & .147 & .773 & .517 & .662 & -.170 \\
5 & .25 & .544 & 1.079 & .201 & .765 & .623 & .713 & .052 \\
6 & .50 & .566 & 1.047 & .218 & .744 & .721 & .755 & .247 \\
\hline
\end{tabular}

and restriction of range resulted in $\hat{\theta}_{2}$ becoming more like $\theta_{1}$. It is also possible that the attempt to distinguish dimensions based on âs for the items pure on $\theta_{1}$ did not align the true and obtained dimensions.

In summary, a correlation between the $\theta$ dimensions seemed to result in slightly poorer estimation for $\theta_{1}$ in all datasets. For $\theta_{2}$, a correlation between dimensions resulted in better estimation for all datasets. A correlation between the $\theta$ dimensions and restriction of range resulted in $\hat{\theta}_{2}$ becoming more like $\theta_{1}$. It seemed to be more difficult for the program to distinguish between the dimensions and there was a greater tendency to collapse the space.

\section{Item Parameter Estimates}

The maximum likelihood estimation procedures in MIRTE use $\theta$ estimates to improve item parameter estimates and vice versa. Hence, the final estimates are affected by each other. Thus, it was of interest to examine the effects of increases in $\rho\left(\theta_{1}, \theta_{2}\right)$ and restriction of range for $\theta_{2}$ on the item parameter estimates.

Statistics on the item difficulty parameters are summarized in Table 3. In Datasets 1-3, $r(d, \hat{d})=.997$, which indicated good recovery of $d$. As $\rho\left(\theta_{1}, \theta_{2}\right)$ increased, the mean and SD of $d$ were increasingly overestimated but remained close to the original parameters. In Datasets 1-3, $\operatorname{AAD}(\hat{d})$ increased slightly as the correlation between $\theta$ dimensions increased, which indicated that $d$ was being less well recovered. However, the $\mathrm{SE}$ of $d$ decreased as $\rho\left(\theta_{1}, \theta_{2}\right)$ increased. The mean and SD of $D$ were recovered well, although again $D$ was less well recovered as $\rho\left(\theta_{1}, \theta_{2}\right)$ increased. $D$ is a function of the discrimination parameters, and its estimate is therefore affected by the estimates of the $a$ parameters.

Both $d$ and $D$ were less well recovered in Datasets 4-6 than in Datasets 1-3. The rescaling of $\theta_{2}$ to mean 0 and SD 1 resulted in larger estimates for $\theta_{2}$, which suggested that the simulees in Datasets 4-6 were more able than they actually were. In these datasets, there were larger SES for $\hat{d}$ and larger SDs for $\hat{d}$ and $\hat{D}$ than in the other datasets. As $\rho\left(\theta_{1}, \theta_{2}\right)$ increased, $\operatorname{SE}(\hat{d})$ decreased but $\operatorname{AAD}(\hat{d})$ increased. However, the recovery of the relationships between the parameters and their estimates remained high $-r(d, \hat{d})>.98$ and $r(D, \hat{D})>.95$-and there was little change in these correlations as $\rho\left(\theta_{1}, \theta_{2}\right)$ increased. It is

Table 3

Means Over 100 Replications of Statistics for Item Difficulty

\begin{tabular}{ccccccccc}
\hline \hline Dataset & $\hat{d}$ & $\mathrm{SD}(\hat{d})$ & $\mathrm{SE}(\hat{d})$ & $\mathrm{AAD}(\hat{d})$ & $\hat{D}$ & $\mathrm{SD}(\hat{D})$ & $r(d, \hat{d})$ & $r(D, \hat{D})$ \\
\hline True & .009 & 3.771 & & & -.005 & 2.058 & & \\
1 & .009 & 3.929 & .112 & .224 & .006 & 2.079 & .997 & .995 \\
2 & .010 & 3.936 & .109 & .228 & .005 & 2.028 & .997 & .994 \\
3 & .030 & .936 & .106 & .232 & .012 & 1.999 & .997 & .991 \\
4 & -.726 & 3.995 & .137 & .811 & .460 & 2.535 & .984 & .958 \\
5 & -.734 & 4.001 & .132 & .827 & .434 & 2.459 & .982 & .956 \\
6 & -.716 & 4.044 & .123 & .834 & .397 & 2.247 & .982 & .969 \\
\hline
\end{tabular}


interesting to note the high correlation of $D$ with $\hat{D}$ because $D$ is a function of $A$ (which was not well recovered) and $d$. Obviously the range of $d$ $(-6$ to +6$)$ affected estimates of $D$ much more than did the two values (2.00 and 1.70) of the $A$ parameters.

Discrimination parameter estimates were less well recovered than difficulty parameter estimates. The mean of $\hat{a}_{1}$ was lower than the true mean, and its SD was higher than the true SD for all six datasets (see Table 4). The means of $\hat{a}_{2}$ were much higher than the true mean of .678 . In fact, the mean of $\hat{a}_{2}$ was higher than the mean estimates of $a_{1}$ and approached the true mean of $a_{1}$ as $\rho\left(\theta_{1}, \theta_{2}\right)$ increased. The means of $\hat{a}_{2}$ and $\hat{a}_{1}$ both increased slightly as $\rho\left(\theta_{1}, \theta_{2}\right)$ increased. The SD of $\hat{a}_{2}$ was higher than the true SD but there was not as large a difference as with $\hat{a}_{1}$. SEs of estimation of $\hat{a}_{1}$ and $\hat{a}_{2}$ were approximately .09 for Datasets 1-3, but were somewhat higher for Datasets 4-6.

For Datasets 4-6, the SEs for $\hat{a}_{2}$ were smaller than for $\hat{a}_{1}$. The SEs for $\hat{a}_{2}$ tended to decrease as $\rho\left(\theta_{1}, \theta_{2}\right)$ increased, but the SEs for $\hat{a}_{1}$ increased. For Datasets 1-3, the $\operatorname{AAD}\left(\hat{a}_{2}\right)$ was much larger than $\operatorname{AAD}\left(\hat{a}_{1}\right)$. For Datasets 4-6 it was only slightly larger.

$A$ was recovered with a higher mean and higher SD in Datasets 1-3. Over the 100 replications, the mean $\hat{A}$ was closer to the true value for Datasets 4-6; however, within replications it was much more variable than for Datasets 1-3.

There appeared to be a rotational indeterminacy in the recovery of each of the $a$ parameters and a tendency to spread the $a$ parameter esti- mates over the entire space even though they originally did not cover the entire space. This was supported by the estimates of the angles $\alpha_{1}$ and $\alpha_{2}$ (the complement of $\alpha_{1}$ ). $\alpha_{1}$ had a mean of $22.50^{\circ}$. This mean was recovered in all datasets at over $49^{\circ}$. Similarly, the mean of $\alpha_{2}$ was $67.50^{\circ}$, and it was recovered in all datasets at just over $40^{\circ}$. The original SD of $16.85^{\circ}$ increased for the estimates to approximately $20^{\circ}$. Estimates of $\alpha_{1}$ and $\alpha_{2}$ ranged from very close to $0^{\circ}$ to almost $90^{\circ}$. For all parameters relating to discrimination $\left(a_{1}, a_{2}\right.$, $\left.A, \alpha_{1}, \alpha_{2}\right)$, their estimates seemed to cover the entire $\theta_{1}, \theta_{2}$ space.

Table 5 shows parameter recovery of the discrimination parameters as correlations. In all cases, $a_{1}$ correlated more highly with $\hat{a}_{1}$ than with $\hat{a}_{2}$, and $a_{2}$ correlated more highly with $\hat{a}_{2}$ than $a_{1}$ did with $\hat{a}_{2} . \hat{a}_{2}$ correlated more highly with $a_{2}$ than $a_{1}$ did with $\hat{a}_{1}$ (except for Dataset 4 ). $a_{2}$ correlated more highly with $\hat{a}_{2}$ than it did with $\hat{a}_{1}$ (except for Dataset 4). The anomaly in the correlations was the similarity of the relationship of $a_{1}$ and $a_{2}$ with $\hat{a}_{1}$. The correlation between $A$ and its estimate was low $(<.6)$ in Datasets $1-3$ and extremely low $(<.3)$ in Datasets 4-6. The high correlation between $\alpha_{1}$ and its estimate for Datasets 1-3 was not expected after obtaining such poor estimates of the multivariate discrimination parameters. $r\left(\alpha_{1}, \hat{\alpha}_{1}\right)$ was much smaller in Datasets 4-6.

Restriction of range of the second trait, $\theta_{2}$, drastically affected the recovery of the discrimination parameters $\left(a_{1}, a_{2}\right.$, and $\left.A\right)$ in Datasets 4-6. For all three variables, the squared correlations between the parameter and its estimate indicate that they had less than half as much variance

Table 4

Means Over 100 Replications of Statistics for Item Discrimination

\begin{tabular}{cccccccccccc}
\hline \hline Dataset & $\hat{a}_{1}$ & $\operatorname{SD}\left(\hat{a}_{1}\right)$ & $\mathrm{SE}\left(\hat{a}_{1}\right)$ & $\mathrm{AAD}\left(\hat{a}_{1}\right)$ & $\hat{a}_{2}$ & $\operatorname{SD}\left(\hat{a}_{2}\right)$ & $\mathrm{SE}\left(\hat{a}_{2}\right)$ & $\operatorname{AAD}\left(\hat{a}_{2}\right)$ & $\hat{A}$ & $\operatorname{SD}(\hat{A})$ & $\hat{\alpha}_{1}$ \\
\hline True & 1.637 & .251 & & & .678 & .496 & & & 1.850 & .151 & $22.50^{\circ}$ \\
1 & 1.195 & .569 & .099 & .500 & 1.379 & .512 & .096 & .707 & 1.957 & .288 & $49.07^{\circ}$ \\
2 & 1.201 & .528 & .095 & .486 & 1.448 & .582 & .094 & .775 & 2.013 & .319 & $49.40^{\circ}$ \\
3 & 1.202 & .502 & .093 & .490 & 1.510 & .628 & .093 & .836 & 2.057 & .381 & $49.98^{\circ}$ \\
4 & 1.076 & .551 & .119 & .623 & 1.228 & .449 & .112 & .653 & 1.736 & .398 & $49.09^{\circ}$ \\
5 & 1.094 & .557 & .138 & .620 & 1.298 & .501 & .108 & .708 & 1.803 & .449 & $49.76^{\circ}$ \\
6 & 1.139 & .599 & .134 & .624 & 1.408 & .534 & .103 & .791 & 1.922 & .495 & $50.94^{\circ}$ \\
\hline
\end{tabular}


Table 5

Mean Correlations (Over 100 Replications) for Item Discriminations

\begin{tabular}{cccccccc}
\hline \hline Dataset & $r\left(a_{1}, \hat{a}_{1}\right)$ & $r\left(a_{2}, \hat{a}_{2}\right)$ & $r\left(\hat{a}_{1}, \hat{a}_{2}\right)$ & $r\left(a_{1}, \hat{a}_{2}\right)$ & $r\left(a_{2}, \hat{a}_{1}\right)$ & $r(A, \hat{A})$ & $r\left(\alpha_{1}, \hat{\alpha}_{1}\right)$ \\
\hline True & - & - & -.738 & - & - & - & - \\
1 & .834 & .893 & -.765 & -.572 & -.865 & .600 & .943 \\
2 & .818 & .899 & -.769 & -.587 & -.830 & .565 & .933 \\
3 & .760 & .895 & -.735 & -.587 & -.747 & .502 & .907 \\
4 & .530 & .523 & -.428 & -.309 & -.543 & .296 & .630 \\
5 & .460 & .511 & -.401 & -.306 & -.455 & .269 & .586 \\
6 & .431 & .514 & -.459 & -.285 & -.403 & .285 & .564 \\
\hline
\end{tabular}

in common for Datasets 4-6 than for Datasets 1-3.

\section{Discussion and Implications}

This study was designed to determine how well multidimensional IRT trait and item parameters would be estimated under different degrees of correlation between the two trait dimensions and the existence of a restricted trait on the second dimension. The best estimates of $\theta_{1}$ and the poorest estimates of $\theta_{2}$ occurred at 0 correlation between traits. This relationship was shown in two ways - the size of the AADs and the correlation of each $\hat{\theta}$ with its parameter. Except for $\operatorname{AAD}\left(\hat{\theta}_{2}\right)$, the same pattern was found for Datasets 4-6 in which $\theta_{2}$ had a restricted range.

The $\theta_{2}$ estimates were affected by the rescaling of the estimates to a 0,1 distribution at each iteration. Evidence of this is that values of $\operatorname{AAD}\left(\hat{\theta}_{2}\right)$ were almost double those in the restricted datasets. With a restricted trait on the second dimension, traits were not as well estimated. As the correlation between dimensions increased, $\hat{\theta}_{2}$ became more highly correlated with $\boldsymbol{\theta}_{1}$, and with restriction of range these correlations exceeded those of $\hat{\theta}_{2}$ with its parameter. Thus, it seems that as traits become more highly correlated and the range of $\theta$ is restricted there is a tendency for the space to collapse and become more unidimensional.

The difficulty parameters were well recovered in all datasets. SEs of $\hat{d}$ were small, and correlations of both $d$ and $D$ with their estimates were quite high $(r \geq .956)$. The AADs for the restricted trait datasets (Datasets 4-6) were quite large, which resulted from forcing $\theta$ s to have a
0,1 distribution. Thus, the data indicate that recovery of multidimensional difficulties was not greatly affected by correlated traits.

The recovery of discrimination parameters was not good. There seemed to be a tendency for $\theta_{2}$ to become the dominant dimension. This was evidenced by the mean $\hat{a}$ and the mean $\hat{\alpha} s$. The variance of $a_{2}$ in the original items was almost four times as great as that for $a_{1}$. This seemed to be a likely explanation of the dominance of $\theta_{2}$. The estimates also seemed to be scattered throughout the space.

Carlson (1987) reported that estimates of $a$ parameters are sensitive to their distribution in the generated data. In the present study, the $a_{i}$ parameters were not distributed over the entire latent space. In addition, the variance of the $a_{2}$ parameters was almost four times as large as for the $a_{1}$ parameters. This restricted the recovery of the $a$ parameters which, in turn, affected the recovery of the $\theta$ and $d$ parameters. It is also possible that MIRTE might not function as well as previous pilot testing had suggested.

\section{Some Problems}

Rescaling of the $\theta_{2}$ estimates. The rescaling of the $\theta_{2}$ estimates in the restricted datasets seemed to affect estimates of difficulty as well as estimates of $\theta s$ and discriminations. Perhaps they should have been rescaled to the mean and SD of the true $\theta_{2}$, but this would require having information that would not be known with nonsimulated data. Thus, operation of MIRTE was examined here as well as the concept of a restricted $\theta_{2}$ trait. The $\hat{d}$ and $\hat{D}$ means were adversely affected in the restricted datasets. However, 
correlations between $d$ and $\hat{d}$, and $D$ and $\hat{D}$ were high $(r>.95)$. The estimates of the $a_{2}$ means improved in these datasets. From the results reported here, the extent of the effects of rescaling cannot be determined, but it appears that the rescaling problem affects all parameter estimates.

Recovery of the two-dimensional space. There was a tendency for the space to collapse as the traits became more correlated. Recovery of the structure of the trait space may relate to a rotational indeterminacy in the recovery of the traits. Correlations of $\theta_{1}$ with $\hat{\theta}_{1}$ were moderately high $(r>.744)$ for the six datasets. This would indicate that $\theta_{1}$ had been recovered relatively well. However, as $\rho\left(\theta_{1}, \theta_{2}\right)$ increased, the correlation between $\theta_{1}$ and $\hat{\theta}_{2}$ increased and, in Dataset 6, $r\left(\theta_{1}, \hat{\theta}_{2}\right)>r\left(\theta_{1}, \hat{\theta}_{1}\right)(.755$ vs. 744). This higher correlation of $\theta_{1}$ with $\hat{\theta}_{2}$ suggests that $\hat{\theta}_{1}$ was off by a rotational factor. This would, in part, explain the AAD for Dimension 1 and the modest correlation between $\theta_{1}$ and $\hat{\theta}_{1}$. This correlation may underestimate the recovery of $\theta_{1}$ in the twodimensional space. The correlations of $\theta_{2}$ and $\hat{\theta}_{1}$ were low, which indicated that $\theta_{2}$ was recovered at approximately the level indicated by the correlation between $\theta_{2}$ and $\hat{\theta}_{2}$. The rotational issue is important in considering whether a dimension is recovered poorly or matched poorly in the particular rotation. Dimension 2 was recovered modestly whereas Dimension 1 was recovered far better, but the rotational position of $\hat{\theta}_{1}$ masked this fact.

In the initial research design, some items pure on Dimension 2 were included to anchor the traits in an attempt to improve the recovery of all parameters. Because such a test would not simulate the desired conditions, these items were not included. This might be reconsidered in a future design.

The collapsing of the space as $\rho\left(\hat{\theta}_{1}, \theta_{2}\right)$ increased not only affected the $\theta$ estimates but also the discrimination estimates. In Datasets 4-6, the structure of the latent space was recovered less well than in the other datasets. In retrospect, combining corresponding datasets of the two types prior to analysis of the number-correct score vectors would provide a sample that might more typically represent the situation in which ESL students would likely be placed and would have allowed for better coverage of the $\theta_{1}, \theta_{2}$ space. This might improve the estimation of some parameters and it would also reduce the effects of the problems caused by the rescaling of $\hat{\theta}_{2}$.

Dimensionality of the item space. 26 of the items were unidimensional (pure on Dimension 1). The remaining 78 were two-dimensional- 52 required primarily $\theta_{1}$, and 26 required equal amounts of $\theta_{1}$ and $\theta_{2}$. The latent structure of the data was more complex than a two-dimensional test composed of two sets of unidimensional items. There were serious concerns with respect to the recovery of the item space-the most serious being the apparent dominance of $\hat{\theta}_{2}$ over $\hat{\theta}_{1}$, or $\hat{\alpha}_{2}$ over $\hat{\alpha}_{1}$. The poor recovery of the discrimination parameters also affected recovery of the difficulty and trait parameters. The item space seemed to become somewhat unidimensional. The estimates of the $a_{i}$ s were more alike and the size of the $\alpha$ angle estimates moved toward $45^{\circ}$ with $\hat{\alpha}_{2}$ becoming dominant. Because the range of $a_{2}$ was greater than that of $a_{1}$, this could have affected the dominance of $\hat{a}_{2}$ over $\hat{a}_{1}$.

Interpretation of parameter estimates appears to depend on the model, $\rho\left(\theta_{1}, \theta_{2}\right)$, and the characteristics of the dataset. From the results presented here, there is every indication that there are indeed three components of multidimensionality (examinee dimensionality, test dimensionality, and the interaction of the two) as suggested by McKinley \& Reckase (1984). Although the population may be multidimensional, if the test is largely unidimensional, resulting scores will tend to unidimensionality as well. It may be expecting too much of the model and MIRTE to have better recovery of the parameters relating to the second dimension when few items measured that dimension and when the populations in the restricted datasets were low on trait level in the second dimension. As a result, little information could be obtained on items of higher difficulty measuring the second dimension. 


\section{Future Directions}

Several questions remain that suggest future studies. First, are the results affected by the estimation procedures and/or the model selected? Replication of the research using different models [perhaps the multidimensional three-parameter logistic IRT model of Bogan \& Yen (1983) or a noncompensatory model] would indicate the extent to which the model affected results. Inclusion of a guessing parameter in the model also would provide additional information. A version of MIRTE (Carlson, 1987) allows for inclusion of the guessing parameter.

It also would be useful to estimate item parameters only while holding the given trait parameters fixed and vice versa to determine further the efficiency of MIRTE. These results could be compared with those obtained when item and trait parameters are estimated simultaneously. Presumably, both item and trait parameters would be better estimated when the other parameter is held fixed.

Corresponding restricted and nonrestricted datasets could be combined in a suitable ratio to present the ESL group as part of a large sample of non-ESL examinees. This would be more typical of real data. This should solve some of the rescaling and space problems.

The test design could be altered to allow for better distribution of the discrimination parameters. The discrimination and difficulty parameters also could be generated randomly to cover the space. The test would then not simulate the condition that it primarily measure one of the two dimensions. However, valuable information could be gained on parameter recovery.

It would be useful to determine the means of trait estimates at different trait levels rather than just reporting the mean level over all trait levels. This could be ascertained by examining the $\theta$ vectors in different sections of the $\theta_{1}, \theta_{2}$ space and comparing the original $\theta$ s with $\theta$ estimates. It also would be useful to know how influential the second trait dimension becomes as the items require more of this trait for a correct response.
An important finding of this research is the capability of MIRTE to retain the structure of the data and the examinees. Although there was some tendency to collapse the latent space as $\rho\left(\theta_{1}, \theta_{2}\right)$ increased, estimates provided by MIRTE recovered two dimensions. It would be appropriate to further develop estimation programs so that rotational solutions could be produced that might alleviate the tendency to collapse a twodimensional space as the correlation between the dimensions increases.

\section{Conclusions}

When a compensatory model is used, correlated dimensions affect recovery both of the dominant and less dominant dimensions. Restriction of range seems also to affect recovery. This seems likely even without a rescaling of the $\theta$ s to 0,1 . Of the parameter estimates, difficulty is least affected. The fact that the difficulty estimates are influenced by multiple trait and discrimination estimates may assure that difficulty is well estimated.

\section{References}

Ackerman, T. A. (1985). M2PLGEN: A computer program for generating thetas and response strings corresponding to the M2PL model. Iowa City IA: American College Testing.

Ackerman, T. A. (1987, April). The use of unidimensional item parameter estimates of multidimensional items in adaptive testing. Paper presented at the annual meeting of the American Educational Research Association, Washington D.C.

Ansley, T. N., \& Forsyth, R. A. (1985). An examination of the characteristics of unidimensional IRT parameter estimates derived from two-dimensional data. Applied Psychological Measurement, 9, 37-48.

Bogan, E. D., \& Yen, W. M. (1983, April). Detecting multidimensionality and examining its effects on vertical equating with the three-parameter logistic model. Paper presented at the annual meeting of the American Educational Research Association, Montreal, Canada.

Carlson, J. E. (1987). Multidimensional item response theory estimation: A computer program (ACT Research Rep. No. 87-19). Iowa City IA: American College Testing Program.

Dorans, N. J., \& Kingston, N. M. (1985). The effects of violations of unidimensionality on the estima- 
tion of item and ability parameters and on item response theory equating of the GRE verbal scale. Journal of Educational Measurement, 22, 249-262.

Drasgow, F., \& Parsons, C. K. (1983). Application of unidimensional IRT models to multidimensional data. Applied Psychological Measurement, 7, 189-199.

Greaud, V. A. (1988, April). Some effects of applying unidimensional IRT to multidimensional tests. Paper presented at the annual meeting of the American Educational Research Association, New Orleans.

International Mathematical and Statistical Libraries. (1979). IMSL Library (7th ed.). Houston TX: Author.

McCauley, C. D., \& Mendoza, J. (1985). A simulation study of item bias using a two-parameter item response model. Applied Psychological Measurement, 9, 389-400.

McKinley, R. L. (1983, April). A multidimensional extension of the two-parameter logistic latent trait model. Paper presented at the annual meeting of the $\mathrm{Na}$ tional Council on Measurement in Education, Montreal, Canada. (ERIC Document Reproduction Service No. ED 228 326)

McKinley, R. L., \& Reckase, M. D. (1983a). An application of a multidimensional extension of the twoparameter logistic latent trait model (ONR-83-3). (ERIC Document Reproduction Service No. ED $240168)$

McKinley, R. L., \& Reckase, M. D. (1983b). MAXLOG: A computer program for the estimation of the parameters of a multidimensional logistic model. Behavior Research Methods and Instrumentation, 15, 389-390.

McKinley, R. L., \& Reckase, M. D. (1984). An investi- gation of the effect of correlated abilities on observed test characteristics (Research Report). Iowa City IA: American College Testing Program, Test Development Division. (ERIC Document Reproduction Service No. ED 249 249)

Muraki, E., \& Englehard, G. (1985). Full-information item factor analysis: Applications of EAP scores. Applied Psychological Measurement, 9, 417-430.

Reckase, M. D. (1979). Unifactor latent trait models applied to multifactor tests: Results and implications. Journal of Educational Statistics, 4, 207-230.

Reckase, M. D. (1985). The difficulty of test items that measure more than one ability. Applied Psychological Measurement, 9, 401-412.

Reckase, M. D., Carlson, J. E., Ackerman, T. A., \& Spray, J. A. (1986, June). The interpretation of unidimensional IRT parameters when estimated from multidimensional data. Paper presented at the annual meeting of the Psychometric Society, Toronto, Ontario.

Reckase, M. D., \& McKinley, R. L. (1991). The discriminating power of items that measure more than one dimension. Applied Psychological Measurement, 15, 361-373.

Wingersky, M. S., Barton, M. A., \& Lord, F. M. (1982). LOGIST user's guide. Princeton NJ: Educational Testing Service.

\section{Author's Address}

Send requests for reprints or further information to Marvin W. Boss, Faculty of Education, University of Ottawa, 145 Jean-Jacques Lussier, Ottawa, Ontario K1N 6N5, Canada. 